\title{
THEORETICAL ANALYSIS OF COMPLEX-CONJUGATE-AMBIGUITY SUPPRESSION IN FREQUENCY-DOMAIN OPTICAL-COHERENCE TOMOGRAPHY
}

\author{
S. Chandra Sekhar ${ }^{*}$, Roland Michaely ${ }^{2}$, Rainer A. Leitgeb ${ }^{3}$, and Michael Unser ${ }^{4}$ \\ ${ }^{1,4}$ Biomedical Imaging Group, ${ }^{2}$ Biomedical Optics Laboratory \\ Ecole Polytechnique Fédérale de Lausanne (EPFL), Switzerland \\ ${ }^{3}$ Center for Biomedical Engineering and Physics \\ Medical University of Vienna, Austria \\ Emails: \{chandrasekhar.seelamantula, roland.michaely, michael.unser\} @epfl.ch, \\ rainer.leitgeb@meduniwien.ac.at
}

\begin{abstract}
New phase-shifting techniques have recently been proposed to suppress the complex-conjugate ambiguity in frequencydomain optical-coherence tomography. A phase shift is introduced, in an elegant fashion, by incorporating a small beam offset at the scanning mirror. The tomogram is then computed by using a combination of Hilbert and Fourier transforms. This is a marked deviation from the conventional approaches, wherein each A-scan is reconstructed independently of the others. In this paper, we formulate the problem in a signal processing framework and provide theoretical proofs for maximal and partial suppression of complex-conjugate ambiguity. To supplement the theoretical derivations, we provide experimental results on in vivo measurements of a human finger nail.
\end{abstract}

Index Terms - frequency-domain optical-coherence tomography, complex-conjugate ambiguity, Hilbert transform, Bedrosian theorem.

\section{INTRODUCTION}

The foundation for frequency-domain optical-coherence tomography (FDOCT) is the inverse scattering theorem, which states that the inverse Fourier transform of the spectral interference pattern yields the axial sample structure $[1,2,3]$. This property enables the reconstruction of depth profiles based on measurements made at the surface without a need for scanning [2]. Tomographic reconstruction is achieved by simple Fourier transformation. Since the measurements are intensities, which are inherently real-valued, their Fourier transforms have Hermitian symmetry. This gives rise to the socalled "complex-conjugate ambiguity". Quite often, the ambiguity is resolved by placing the zero-delay plane of the interferometer outside the specimen. However, this solution has some limitations; for example, the farther the object is

\footnotetext{
* This work is supported by the Hasler foundation.
}

from the zero-delay plane, the poorer is the sensitivity. The best signal quality is achieved when the zero-delay plane lies within the specimen. In principle, this arrangement doubles the accessible depth. The downside of this configuration is the complex-conjugate ambiguity. The commonly employed approaches to overcome this problem are based on phaseshifting interferometry. In this approach, a second set of measurements is acquired, preferably in quadrature phase, and the complex-conjugate function is canceled by a suitable combination of the two measurements. A disadvantage of these approaches is that phase shifters often tend to have chromatic aberrations. The problem is more complicated for in vivo experiments because mild movement of the sample may alter the phase relation. Yet another disadvantage of the phase-shifting approaches is that twice the number of measurements are required for accurate reconstruction.

We briefly review some recently proposed techniques that enable optimal reconstruction. Bachmann et. al [4] introduced a frequency-shifting technique that allows for a complete achromatic heterodyne signal reconstruction. Vakoc et. al. [5] proposed a technique for eliminating depth degeneracy by instantaneously acquiring two phase-shifted signals. Their system employs a passive optical demodulation circuit and a chirped digital acquisition clock derived from a voltagecontrolled oscillator. Although this procedure suppresses the artifacts, the complexity of the system increases. Wang [6] proposed an alternative that eliminates the need to acquire multiple measurements, and showed applications for in vivo imaging, both in real time and at video rate. A drawback of this technique is that it requires an additional device-a piezoelectric stage. This not only makes the system more expensive, but also increases the complexity in terms of the synchronization electronics. In particular, for common-path configurations [7], the reference arm is included in a fibercoupled handheld device, which makes it difficult to add phaseshifting components. Recently, we developed a new system to overcome such limitations [8]. Our approach does not need 
additional phase-shifting devices, and can be implemented easily by incorporating a small offset at the beam-scanning mirror. We reported results on in vivo measurements in a FDOCT system employing a handheld scanner. The reconstruction technique is the same as that in [6]. Embedded in these techniques are some of the fundamental properties of the Fourier and Hilbert transforms. Our objective in this paper is to gain a better understanding of the new method by formulating a signal processing model, which not only predicts the reconstruction quality, but also helps in optimizing the system parameters. We provide experimental results based on in vivo measurements, and compare the optimal reconstruction to the conventional and suboptimal ones.

\section{SIGNAL MODEL}

Consider FDOCT in a Michelson interferometric configuration (see [9] for a schematic of the setup). The measured signal is the light reflected from the object, and is coherently amplified by the reference-arm signal. The measurements are recorded by a spectrometer, as a function of the wavelength $\lambda$. In the standard Fourier transform notation, the measurements take the form

$$
I^{\prime}\left(x, \omega_{z}\right)=S\left(\omega_{z}\right)\left|1+\int_{-\infty}^{+\infty} a(x, z) e^{-\mathrm{j} \omega_{z} z} \mathrm{~d} z\right|^{2}
$$

where $S\left(\omega_{z}\right)$ is the source power spectrum, $\omega_{z}=-\frac{4 \pi n}{\lambda}, n$ being the average refractive index of the specimen, and where $a(x, z)$ is the scattering function. The objective is to recover $a(x, z)$ from $I^{\prime}\left(x, \omega_{z}\right)$. Developing the squares in (1), we have that

$$
\begin{aligned}
I^{\prime}\left(x, \omega_{z}\right)= & S\left(\omega_{z}\right)+S\left(\omega_{z}\right) A\left(x, \omega_{z}\right)+S\left(\omega_{z}\right) A^{*}\left(x, \omega_{z}\right) \\
& +S\left(\omega_{z}\right)\left|A\left(x, \omega_{z}\right)\right|^{2}
\end{aligned}
$$

where $A\left(x, \omega_{z}\right)=\int_{-\infty}^{+\infty} a(x, z) e^{-\mathrm{j} \omega_{z} z} \mathrm{~d} z$. The function $S\left(\omega_{z}\right)$ can be suppressed by subtracting the measured source intensity. Let us assume that $S\left(\omega_{z}\right)\left|A\left(x, \omega_{z}\right)\right|^{2}$ is negligible in comparison to the other terms, and that the source coherence function can be approximated by a Dirac impulse. The latter assumption only simplifies the calculations, and does not affect our conclusions. The signal of interest in (2) is therefore

$$
I\left(x, \omega_{z}\right)=A\left(x, \omega_{z}\right)+A^{*}\left(x, \omega_{z}\right) .
$$

Without loss of generality, we assume that $a(x, z)$ is real. The inverse Fourier transform of $I\left(x, \omega_{z}\right)$, calculated along $\omega_{z}$ is given by

$$
i(x, z)=a(x, z)+a(x,-z),
$$

which is the sum of two functions with disjoint support provided that the zero-delay plane is outside of the specimen. In the scenario considered here, the zero-delay plane is inside the specimen; therefore, $a(x, z)$ is not causal along $z$ and the complex-conjugate ambiguity in $i(x, z)$ cannot be resolved.

Next, let us consider the scenario described in [8], where a phase shift is introduced by incorporating a small offset at the beam-scanning mirror. The resulting effect is best described as phase modulation, and often a linear one, provided that the offset is small. The measurements in the new scenario can be expressed as

$$
I\left(x, \omega_{z}\right)=2 \int_{-\infty}^{+\infty} a(x, z) \cos \left(\omega_{z} z+\phi(x)\right) \mathrm{d} z,
$$

where $\phi(x)=\omega_{c} x, \omega_{c}$ being the carrier frequency. Without loss of generality, we set $\phi(0)=0$.

The new approach to retrieve $a(x, z)$ from $I\left(x, \omega_{z}\right)$ is to compute the Hilbert transform of $I$ along $x$, construct an analytic signal, and then apply a Fourier transform along $\omega_{z}$. We next show that this method yields accurate reconstruction only under certain conditions.

\section{THEORETICAL ANALYSIS}

The notation used is as follows. The symbols $\mathscr{F}, \mathscr{F}^{-1}$ and $\mathscr{H}$ denote the Fourier, inverse Fourier, and Hilbert transforms, respectively. Since the signals involved in our analysis are functions of two variables, we use the subscript to denote the variable with respect to which the transformation is performed. For example, the expression $\mathscr{F}_{\omega_{z}}^{-1}\{A\}(x, z)$ denotes the inverse Fourier transform of $A$ calculated along $\omega_{z}$; the result is a function of the two variables $x$ and $z$. To indicate even and odd functions, we use the subscripts $e$ and $o$, respectively. With the help of this notation, $A\left(x, \omega_{z}\right)$ can be written as $A\left(x, \omega_{z}\right)=A_{e}\left(x, \omega_{z}\right)+\mathrm{j} A_{o}\left(x, \omega_{z}\right)$, where $A_{e}$ and $A_{o}$ are the cosine and the sine transforms, respectively. The subscript as is used to denote the analytic version of the corresponding signal.

For the sake of clarity, we consider the cases of optimal and suboptimal reconstruction separately.

Proposition 1. Let $I_{a s}\left(x, \omega_{z}\right)=I\left(x, \omega_{z}\right)+\mathrm{j} \mathscr{H}_{x}\{I\}\left(x, \omega_{z}\right)$. If $\mathscr{F}_{x}\{A\}\left(\omega_{x}, \omega_{z}\right)=0$, for $\omega_{x}>\omega_{c}$, then $\mathscr{F}_{\omega_{z}}^{-1}\left\{I_{a s}\right\}(x, z)=$ $2 e^{\mathrm{j} \phi(x)} a(x, z)$.

Proof. We rewrite (5) as

$I\left(x, \omega_{z}\right)=2 \cos \phi(x) A_{e}\left(x, \omega_{z}\right)-2 \sin \phi(x) A_{o}\left(x, \omega_{z}\right)$.

Consider the Hilbert transform of $I\left(x, \omega_{z}\right)$ calculated along $x$ :

$$
\begin{aligned}
\mathscr{H}_{x}\{I\}\left(x, \omega_{z}\right)= & 2 \mathscr{H}_{x}\left\{\cos \phi A_{e}\right\}\left(x, \omega_{z}\right) \\
& +2 \mathscr{H}_{x}\left\{\sin \phi A_{o}\right\}\left(x, \omega_{z}\right) .
\end{aligned}
$$

To proceed further, we use a property related to the Hilbert transform of the product of two functions, popularly known 
as the Bedrosian theorem [10]. The theorem states that if two functions $f(x)$ and $g(x)$ have lowpass and highpass spectra, respectively, and they are nonoverlapping, then

$$
\mathscr{H}_{x}\{f g\}(x)=f(x) \mathscr{H}_{x}\{g\}(x) ;
$$

that is, the lowpass function can be factored out of the Hilbert transform operation.

Since $\mathscr{F}_{x}\{A\}\left(\omega_{x}, \omega_{z}\right)=0$, for $\omega_{x}>\omega_{c}, A_{e}$ and $A_{o}$ also satisfy this property. Therefore, the Bedrosian theorem is applicable in (7), which then reduces to

$$
\begin{aligned}
\mathscr{H}_{x}\{I\}\left(x, \omega_{z}\right)= & 2 \sin \phi(x) A_{e}\left(x, \omega_{z}\right) \\
& +2 \cos \phi(x) A_{o}\left(x, \omega_{z}\right) .
\end{aligned}
$$

The corresponding analytic signal $I_{a s}\left(x, \omega_{z}\right)$ is given by

$$
\begin{aligned}
I_{a s}\left(x, \omega_{z}\right) & =2 e^{\mathrm{j} \phi(x)}\left(A_{e}\left(x, \omega_{z}\right)+\mathrm{j} A_{o}\left(x, \omega_{z}\right)\right) \\
& =2 e^{\mathrm{j} \phi(x)} A\left(x, \omega_{z}\right) .
\end{aligned}
$$

From (10), we have that

$$
\mathscr{F}_{z}^{-1}\left\{I_{a s}\right\}(x, z)=2 e^{\mathrm{j} \phi(x)} a(x, z) .
$$

Thus, we have shown that $a(x, z)$ can be determined unambiguously from $I\left(x, \omega_{z}\right)$, provided that $\mathscr{F}_{x}\{A\}\left(\omega_{x}, \omega_{z}\right)=$ 0 for $\omega_{x}>\omega_{c}$. Next, we consider the case where this condition is violated.

Proposition 2. If $\mathscr{F}_{x}\{A\}\left(\omega_{x}, \omega_{z}\right) \neq 0$ for $\omega_{x}>\omega_{c}$, then $a(x, z)$ cannot be determined uniquely from $I\left(x, \omega_{z}\right)$.

Proof. Let $A_{e}=A_{e_{1}}+A_{e_{2}}$ such that $\mathscr{F}_{x}\left\{A_{e_{1}}\right\}\left(\omega_{x}, \omega_{z}\right)=0$, for $\omega_{x}>\omega_{c}$, and $\mathscr{F}_{x}\left\{A_{e_{2}}\right\}\left(\omega_{x}, \omega_{z}\right)=0$, for $\omega_{x}<\omega_{c}$. Consider a similar lowpass-highpass decomposition for $A_{o}$; i.e., $A_{o}=A_{o_{1}}+A_{o_{2}}$. In terms of these functions, we rewrite (6) as

$$
\begin{aligned}
I\left(x, \omega_{z}\right)= & 2 \cos \phi(x)\left(A_{e_{1}}\left(x, \omega_{z}\right)+A_{e_{2}}\left(x, \omega_{z}\right)\right) \\
& -2 \sin \phi(x)\left(A_{o_{1}}\left(x, \omega_{z}\right)+A_{o_{2}}\left(x, \omega_{z}\right)\right) .
\end{aligned}
$$

The Hilbert transform of $I\left(x, \omega_{z}\right)$ computed along $x$ is

$$
\begin{aligned}
\mathscr{H}_{x}\{I\}\left(x, \omega_{z}\right)= & 2 \sin \phi(x) A_{e_{1}}\left(x, \omega_{z}\right) \\
& +2 \cos \phi(x) \mathscr{H}_{x}\left\{A_{e_{2}}\right\}\left(x, \omega_{z}\right) \\
& +2 \cos \phi(x) A_{o_{1}}\left(x, \omega_{z}\right) \\
& -2 \sin \phi(x) \mathscr{H}_{x}\left\{A_{o_{2}}\right\}\left(x, \omega_{z}\right) .
\end{aligned}
$$

Note that we have used the Bedrosian theorem in arriving at the above equation. The corresponding analytic signal $I_{a s}\left(x, \omega_{z}\right)$ is given by

$$
\begin{aligned}
I_{a s}\left(x, \omega_{z}\right)= & 2 e^{\mathrm{j} \phi(x)}(\underbrace{A_{e_{1}}\left(x, \omega_{z}\right)+\mathrm{j} A_{o_{1}}\left(x, \omega_{z}\right)}_{A_{1}\left(x, \omega_{z}\right)}) \\
& +2 \cos \phi(x) A_{e_{2} a s}\left(x, \omega_{z}\right) \\
& -2 \sin \phi(x) A_{o_{2 a s}}\left(x, \omega_{z}\right) .
\end{aligned}
$$

The inverse Fourier transform of (14) is given by

$$
\begin{aligned}
\mathscr{F}_{z}^{-1}\left\{I_{a s}\right\}(x, z)= & 2 e^{\mathrm{j} \phi(x)} a_{1}(x, z)-2 \cos \phi(x) a_{e_{2}}^{-}(x, z) \\
& -2 \sin \phi(x) a_{o_{2}}^{-}(x, z),
\end{aligned}
$$

where $\mathscr{F}^{-1}\left\{A_{1}\right\}(x, z)=a_{1}(x, z)$. The functions that bear the superscript "- " are anti-causal along $z$, and are given by $a_{e_{2}}^{-}(x, z)=\mathscr{F}^{-1}\left\{A_{e_{2}}\right\}(x, z) ; a_{e_{1}}^{-}(x, z)=\mathscr{F}^{-1}\left\{A_{e_{1}}\right\}(x, z)$. Note that $a_{1}(x, z)$ is a two-sided function.

From $(15)$, it is clear that $a(x, z)$ cannot be determined uniquely from $I\left(x, \omega_{z}\right)$.

\section{EXPERIMENTAL RESULTS}

In Figure 1, we show a schematic of the fiber-based FDOCT system developed at the Biomedical Optics Laboratory (EPFL) A broadband light source (LS) is used with the center wavelength at $1300 \mathrm{~nm}$ and a spectral full-width at half-maximum (FWHM) of $70 \mathrm{~nm}$. The axial resolution is $10 \mu \mathrm{m}$ in air. The $70: 30$ fiber coupler transmits $30 \%$ of the light to the samplearm fiber equipped with a handheld device, which contains the adjustable reference arm. The length of the reference arm can be fine-tuned by a translation stage (TS). Within the device, a nonpolarizing beam-splitter is used to divide the light into sample-arm and reference beams. The sample-arm beam is deflected by a single-point two-dimensional scanner and focused via a $50 \mathrm{~mm}$ achromatic lens (L0) into the sample. The spectrometer at the exit of the fiber coupler consists of a collimator (L1), a transmission grating (G) (1200 lines/mm, Wasatch Inc.), and a line array (charge-coupled device, Sensors Unlimited, 512 pixel, 12 bit). The camera objective is an achromatic lens combination (L2, L3) with an effective focal length of $100 \mathrm{~mm}$. The spectrometer resolution is $0.28 \mathrm{~nm}$, allowing for a system depth range of $1.5 \mathrm{~mm}$. The actual exposure time is $22 \mu \mathrm{s}$ with a frame rate of $18.9 \mathrm{kHz}$ or $\mathrm{T}=53 \mu \mathrm{s}$. Corresponding to a power of $2 \mathrm{~mW}$ at the sample, the sensitivity is about $105 \mathrm{~dB}$ close to the zero delay, and it decreases at a rate of $12 \mathrm{~dB} / \mathrm{mm}$. The beam offset is adjusted by a diagonal translation ( $r$ ) of the scanner, which keeps the beam at the center of the focusing optics.

Using the setup described above, we acquired measurements on a finger nail of the first author of this paper, for different values of the phase shift. In Figure 2(a), we show the results obtained by the conventional reconstruction; the difficulty in resolving the complex-conjugate function ambiguity is evident from this figure. This problem is solved quite satisfactorily by using an optimal carrier frequency (cf. Proposition 1, Section 3) as shown in Figure 2(b). In Figure 2(c), we show the reconstructed tomogram corresponding to a suboptimal carrier; in this case, one achieves only partial suppression of the mirror term as predicted by Proposition 2 (Section 3). 


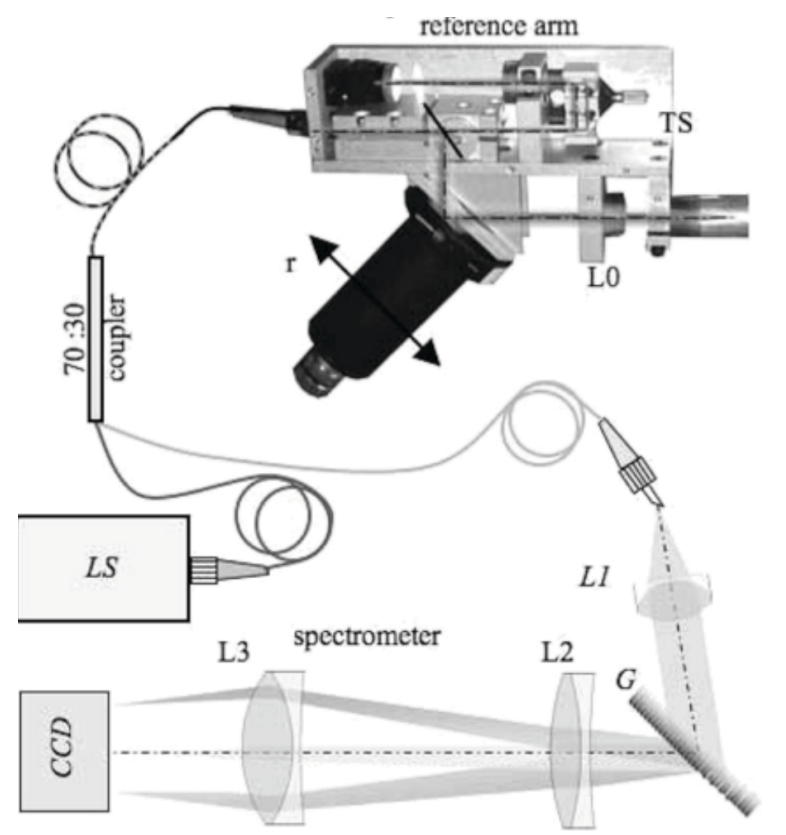

Fig. 1. Schematic of the FDOCT experimental setup employing a handheld scanner. Please refer to Section 4 for explanation.

\section{CONCLUSIONS}

We have addressed the problem of complex-conjugate ambiguity in frequency-domain optical-coherence tomography. We have given theoretical proofs for optimal and suboptimal reconstruction in the context of a newly developed handheld scanning system. The results are also applicable to other phase-shifting techniques. Our analysis is supported by experimental validation on in vivo measurements of a human finger nail. The results show that, by making an optimal choice of the carrier frequency, one can achieve a significant improvement in the reconstruction quality compared with the conventional Fourier technique. In our analysis, we have assumed that the functions are defined on continuous domains. In practice, the effects of sampling must also be taken into account. These results will be reported separately.

\section{REFERENCES}

[1] E. Wolf, "Three-dimensional structure determination of semi-transparent objects from holographic data," Opt. Comm., vol. 1, pp. 153-156, 1969.

[2] A. F. Fercher, C. K. Hitzenberger, G. Kamp, and S. Y. El-Zaiat, "Measurement of intraocular distances by backscattering spectral interferometry," Opt. Comm., vol. 117, pp. 43-48, 1995.

[3] G. Hausler and M. W. Lindner, "Coherence radar and

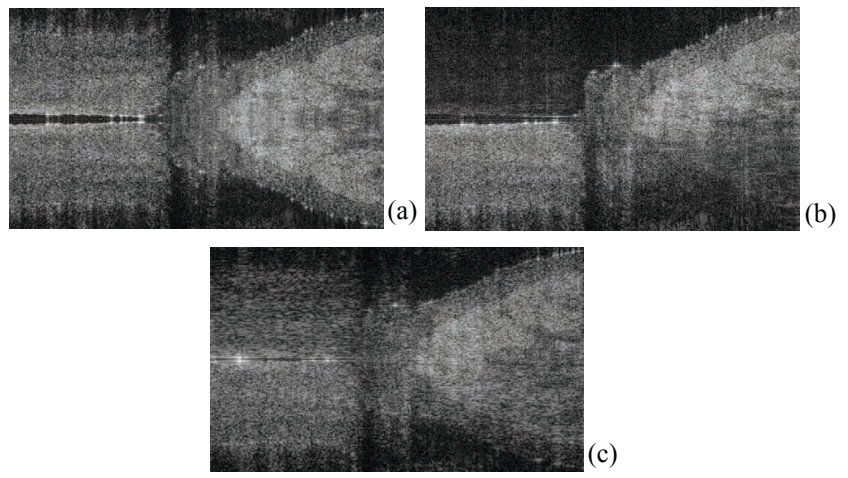

Fig. 2. Experimental results on in vivo measurements of a finger nail: (a) Conventional Fourier reconstruction; Full-range reconstruction by a combination of Hilbert and Fourier transforms: (b) maximal suppression of complex-conjugate ambiguity, and (c) suboptimal reconstruction.

spectral radar-New tools for dermatological analysis," J. Biomed. Opt., vol. 3, pp. 21-31, 1998.

[4] A. H. Bachmann, R. A. Leitgeb, and T. Lasser, "Heterodyne Fourier-domain optical-coherence tomography for full-range probing with high axial resolution," Opt. Exp., vol. 14, no. 4, pp. 1487-1496, 2006.

[5] B. J. Vakoc, S. H. Yun, G. J. Tearney, and B. E. Bouma, "Elimination of depth degeneracy in optical frequencydomain imaging through polarization-based optical demodulation," Opt. Lett., vol. 31, no. 3, pp. 362-364, 2006.

[6] R. K. Wang, "In vivo full range complex Fourier-domain optical-coherence tomography," Appl. Phy. Lett., vol. 90, no. 054103, pp. 1-3, 2007.

[7] A. H. Bachmann, R. Michaely, T. Lasser, and R. A. Leitgeb, "Dual beam heterodyne Fourier-domain opticalcoherence tomography," Opt. Exp., vol. 15, no. 15, pp. 9254-9266, 2007.

[8] R. A. Leitgeb, R. Michaely, T. Lasser, and S. C. Sekhar, "Complex ambiguity-free Fourier-domain optical-coherence tomography through transverse scanning," Opt. Lett., vol. 32, no. 23, pp. 3453-3455, 2007.

[9] S. C. Sekhar, R. A. Leitgeb, M. L. Villiger, A. H. Bachmann, T. Blu, and M. Unser, "Noniterative exact signal recovery in frequency-domain optical-coherence tomography," in Proc. of the Fourth IEEE Intl. Symp. on Biomed. Imaging: From Nano to Macro (ISBI'07), Arlington VA, USA, April 12-15, 2007, pp. 808-811.

[10] E. Bedrosian, "A product theorem for Hilbert transforms," Proc. IEEE, pp. 868-869, 1963. 